\title{
Photonic Processes Visualized with Electrons in Photoemission Electron Microscopy (PEEM)
}

\author{
Rolf Könenkamp $^{1}$, Robert C. Word ${ }^{1}$ and Joseph P. S. Fitzgerald ${ }^{1}$ \\ ${ }^{1}$ Physics Department, Portland State University, Portland, OR 97201, USA
}

Photoemission electron microscopy (PEEM) combines in a unique way light and electron optics: As photo-emitted electrons are used in the imaging process, light-optical phenomena can be imaged with a resolution significantly below the light-optical diffraction limit. In addition the light-optical excitation offers non-destructive operation conditions, an inherent sensitivity to the surface-near region and extremely short observation times [1].

We use aberration-corrected PEEM to directly visualize with electrons the propagation of light at solid surfaces [2]. Aberration-correction is achieved with an electrostatic mirror that allows simultaneous correction of chromatic and spherical aberrations [3]. The typical spatial resolution in this work is 5 to $40 \mathrm{~nm}$. From the PEEM micrographs we can quantitatively determine local optical material properties, phase shifts as well as coupling and scattering probabilities etc. In many cases a detailed quantitative understanding of dynamic optical processes can be achieved. PEEM imaging also allows the direct observation of optical processes in devices such as optical filters, light-routers, plasmonic routers, sensors, etc. [4, 5].

Figure 1 shows the propagation of light waves in a thin, transparent indium tin oxide (ITO) film. Coherent plane wave light from a 100-fs pulsed laser is coupled into the ITO film at a semi-circular arrangement of air holes. The shown image represents an interference pattern resulting from a diffracted light wave in the film and an un-diffracted plane reference wave [6]. As the two waves originate from a pulsed source, the number of interference fringes is finite and the extent and duration of the image are limited: Overall about 100 interference fringes are generated corresponding to a time interval of less than a picosecond. The interaction that gives rise to this electron micrograph is a non-linear 2-photon photoemission process. Careful analysis of this process allows a reconstruction of the image from fundamental optics principles. A two-dimensional image calculation based on Kirchhoff integration is shown in Figure 1c. The analytical image calculation reproduces the experimental features with many details [7]. Beatings between different modes as well as non-linear interactions are seen. Combining the information provided by experiment and simulation allows an accurate determination of film thickness, refractive index, first and secondorder absorption coefficients etc. The high spatial resolution of the electron micrographs also lends itself to a detailed near-field analysis. For example, the polarization dependence of the diffraction process can quantitatively be analyzed in the same microscopy approach [8]. Furthermore, the analysis is not restricted to guided and or confined light modes. Figure 2 shows a photoemission micrograph obtained at a micro-structured silicon surface. A detailed analysis shows that in this case the interferences are generated by diffraction at surface features of the silicon. However, only vacuum modes contribute to the photoelectron emission. We therefore conclude that grazing optical vacuum modes near solid surfaces can produce photoelectron emission and can thus also be used in PEEM for imaging surface properties. Finally it is shown that the same theoretical image analysis can be applied to PEEM micrographs of surface plasmon polariton modes at metal surfaces [9]. 
We conclude that multiphoton-PEEM provides an excellent means to study optical and photonic processes in waveguides and at surfaces. In 2- and 3-photon processes all of the visible range becomes available for photoelectron emission. Spatial resolution significantly below the optical wavelength can be achieved and near-field studies with high time-resolution are feasible.

[1] Ernst Bauer in "Surface Microscopy with Low Energy Electrons", Springer (2014)

[2] Robert C. Word et al., Ultramicroscopy 110, 741 (2010).

[3] Joseph P. S. Fitzgerald et al., Ultramicroscopy 115, 35 (2012)

[4] Rolf Könenkamp et al., Appl. Phys. Lett. 101, 141114 (2012)

[5] Robert C. Word et al., Appl. Phys. Lett. 105, 111114 (2014)

[6] Livio I. Chelaru and F.-J. Meyer zu Heringdorf, Appl. Phys. Lett. 89, 241908 (2006)

[7] J.P.S. Fitzgerald et al., Phys. Rev. B 89, 195129 (2014)

[8] Theodore Stenmark et al., this conference

[9] Niemma M. Buckanie et al., Ultramicroscopy 130, 49 (2013)

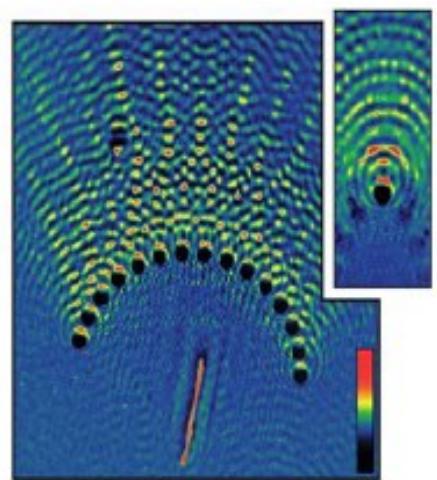

(a)

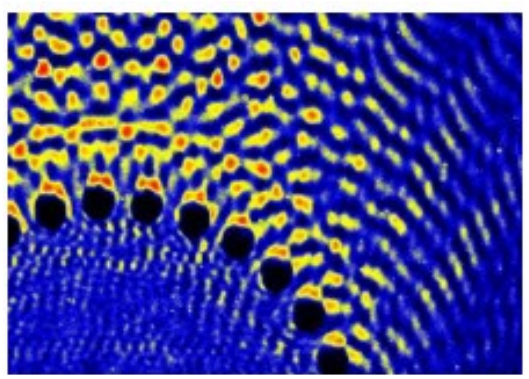

(b)

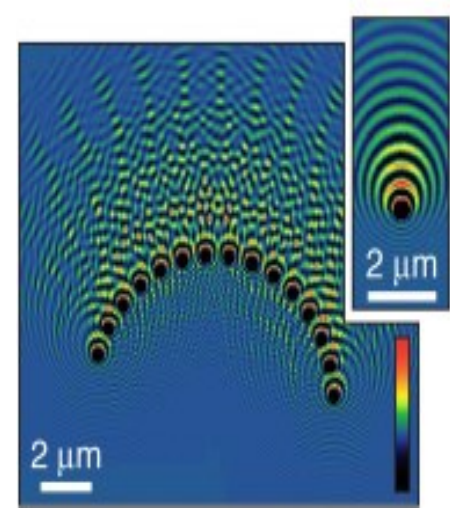

(c)

Figure 1: (a) Experimental PEEM image of a guided optical wave in a thin ITO film. The wave is excited in the 15 holes arranged in a semicircle. A gold nanowire is seen in the lower half of the image. (b) Close-up from the experimental results shown in part (a). (c) Calculated image of the optical system shown in (a). The gold nanowire is not considered in this calculation.

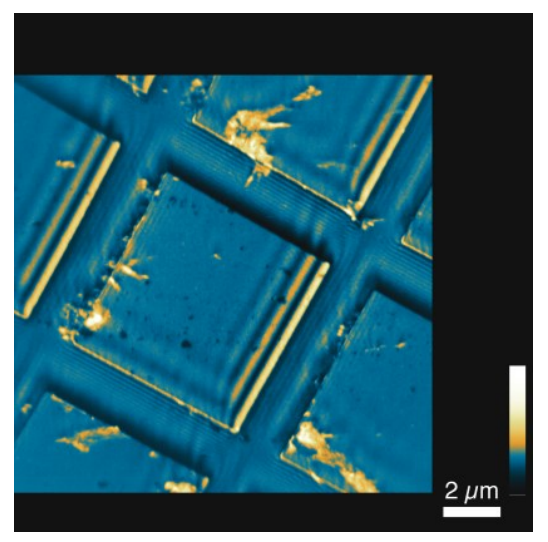

Figure 2: Experimental PEEM image of diffracted vacuum modes generated at a micro-structured silicon surface. 